Immunosuppressive therapy is used in the pathogenetic treatment of rheumatoid arthritis. Methotrexate for rheumatoid arthritis is considered the current gold standard first line treatment. In recent years, the combination of methotrexate and genetic engineering biological drugs is used with high efficiency.

Patient S., 18 years old, was admitted on 24 March 2016 in the rheumatology department of the Amur regional clinical hospital with clinical diagnosis: Rheumatoid arthritis seropositive, deployed stage, activity I, non-erosive (radiographic stage 1), ACCP (-), FCl, complications: secondary amyloidosis with renal impairment, osteoporosis with pathological fractures.

III since 2006, when first appeared pain in the ankle and knee joints. Over the next years the condition worsened, joined by pain in the wrist joints, there was swelling of the knee and limitation of motion in the aforementioned joints. Was examined in the cardiology department of the Amur regional children's clinical hospital. Diagnosis: Juvenile rheumatoid arthritis. Treatment with prednisolone at a dose of $30 \mathrm{mg} /$ day, showed a slight effect. In 2007, the treatment pulse therapy with methylprednisolone $1000 \mathrm{mg} \mathrm{No3}$, cyclophosphamide at a dose of $1000 \mathrm{mg}$ No2 (1 per month), prednisone $1.5 \mathrm{mg} / \mathrm{kg} /$ day. The patient constantly took methotrexate at a dose of $10 \mathrm{mg} / \mathrm{week}$ in combination with folic acid. In 2008, there were pronounced swelling on the face in the periorbital area and on the feet. The examination revealed massive proteinuria, hydrothorax and hydropericardium. Sent to Moscow to Russian children's clinical hospital for further examination, diagnosis and determining further management of patients. Had a biopsy of the kidneys, it results diagnosis: Juvenile rheumatoid arthritis. Secondary renal amyloidosis. In 2012, the RAMS Institute of Rheumatology diagnosis of rheumatoid arthritis, secondary amyloidosis of the kidneys confirmed. Since 2014 receives infliximab at a dose of $200 \mathrm{mg} 1$ every 8 weeks. The present hospitalization is planned in connection with the achievement of the age of eighteen and the transition under the supervision of an adult rheumatologist.

By results of additional methods of examination of clinical, laboratory and radiological disease activity is not marked at the moment. He continues to receive treatment of methotrexate $15 \mathrm{mg}$ per week in combination with folic acid $5 \mathrm{mg}$ per week, infliximab $200 \mathrm{mg} 1$ every 8 weeks via intravenous infusion pump.

The interest of the case lies in the fact that early active basic treatment of juvenile rheumatoid arthritis prevented the progression of destruction of joints, disability of the patient, to neutralize the manifestations of amyloidosis and nephrotic syndrome, improving quality of life in general.

1. Nasonov E.L. Clinical guidelines. Rheumatology. M.: GEOTAR-Media, 2011.-758 p.

2. Nasonov E.L, Nasonova V.A. Rheumatology: National leadership. M.: GEOTAR-Media, 2008.-720 p.

3. Smolen J.S., Lamdewe R., Breedveld F.C. et al. EULAR recommendations for the management of rheumatoid arthritis with synthetic and biological disease-modifying anti-rheumatic drugs. Ann Rheum Dis 2010; 69: 964-975.

DOI 10.22448/AMJ.2017.3.57-57

\title{
THERAPEUTIC EFFECT OF SHIKONIN ON COLLAGEN-INDUCED ARTHRITIS BY DOWN-REGULATION OF TH1 RESPONS- ES AND INDUCTION OF TH2 AND TREG RESPONSES
}

\section{Qiaomei Dai, Meiqiao Wang, Mengting Lu, Hui Liang, Yanqi Shang, Xinxin CHen}

\author{
Department of pathology, Heilongjiang University of Chinese Medicine, Harbin, China
}

\begin{abstract}
Objective: To investigate the therapeutic effect of shikonin on collagen-induced arthritis (CIA) and its influence on Th1/Th2/Treg balance.Methods: Shikonin were given to mouse by oral administration before the onset of $\mathrm{ClA}$, and MTX was used as positive control drug. The arthritis response was monitored visually by macroscopic scoring. Joint swelling were measured by micrometer measurements of mouse. RT-PCR was employed to determine the mRNA expression of cytokines and transcription factors in patella with adjacent synovium in CIA mouse. Histology of knee of CIA was used to assess the occurrence of cartilage destruction and bone erosion. The expression of regulatory $T$ cells on lymph node of CIA was tested by immunohistochemistry. Western blotting was used to detect the expression of FOXP3 in spleen of CIA. Results: Shikonin treatment suppressed the macroscopic score and incidence of arthritis. A more pronounced improvement on the advancement of histology was found in mouse treated with shikonin on $\mathrm{CIA}$, which reduce cellular infiltrates in the synovial tissue and provide protection against cartilage destruction. Swelling of hind paws were delayed with shikonin when compared to controls. Moreover, the expression of Th1 cytokines (IL-18, IL-12, TNF-a) was greatly inhibited both in the synovial tissue and in the articular cartilage in treated groups compared with those in control groups. The mRNA levels of Th2 cytokines(IL-10), however, were up-regulated after shikonin treatment. The expression of factor (Foxp3,TGF-b) in the synovial tissue and in the cartilage was up-regulated after shikonin treatment. T-bet mRNA levels were decreased in shikonin group compared with control group. The expression of Foxp3 protein in spleen was up-regulated after shikonin treatment through Western blotting. The expression of Foxp3 in lymph node has no statistical significance compared with control group through immunohistochemistry. Conclusion: Shikonin has therapeutic effects on collagen induced arthritis. Shikonin treatment can inhibit Th1 cytokines expression and induce Th2 cytokines(IL-10) and TGF-b expression, which may be mediated not only by inhibiting Th1 responses through a T-bet dependent mechanism, but also by inducing cytokins such as IL-10 and TGF-b through a Treg-dependent mechanism.
\end{abstract}

Key words: Shikonin collagen induced arthritis regulatory $T$ cells $T$ helper cells 1 T helper cells 2 